\title{
Association of the Aspartate Aminotransferase to Alanine Aminotransferase Ratio with BNP Level and Cardiovascular Mortality in the General Population: The Yamagata Study 10-Year Follow-Up
}

\author{
Miyuki Yokoyama, ${ }^{1}$ Tetsu Watanabe, ${ }^{1}$ Yoichiro Otaki, ${ }^{1}$ Hiroki Takahashi, ${ }^{1}$ \\ Takanori Arimoto, ${ }^{1}$ Tetsuro Shishido, ${ }^{1}$ Takuya Miyamoto, ${ }^{1}$ Tsuneo Konta, ${ }^{1}$ Yoko Shibata, ${ }^{1}$ \\ Makoto Daimon, ${ }^{2}$ Yoshiyuki Ueno, ${ }^{3}$ Takeo Kato, ${ }^{3}$ Takamasa Kayama, ${ }^{3}$ and Isao Kubota ${ }^{1}$ \\ ${ }^{1}$ Department of Cardiology, Pulmonology, and Nephrology, Yamagata University School of Medicine, Yamagata, Japan \\ ${ }^{2}$ Department of Endocrinology and Metabolism, Hirosaki University Graduate School of Medicine, Aomori, Japan \\ ${ }^{3}$ Global Center of Excellence Program Study Group, Yamagata University School of Medicine, Yamagata, Japan
}

Correspondence should be addressed to Tetsu Watanabe; tewatana@med.id.yamagata-u.ac.jp

Received 10 August 2016; Revised 24 September 2016; Accepted 9 October 2016

Academic Editor: Shih-Ping Hsu

\begin{abstract}
Copyright ( 2016 Miyuki Yokoyama et al. This is an open access article distributed under the Creative Commons Attribution License, which permits unrestricted use, distribution, and reproduction in any medium, provided the original work is properly cited.

Background. Early identification of high risk subjects for cardiovascular disease in health check-up is still unmet medical need. Cardiovascular disease is characterized by the superior increase in aspartate aminotransferase (AST) to alanine aminotransferase (ALT). However, the association of AST/ALT ratio with brain natriuretic peptide (BNP) levels and cardiovascular mortality remains unclear in the general population. Methods and Results. This longitudinal cohort study included 3,494 Japanese subjects who participated in a community-based health check-up, with a 10-year follow-up. The AST/ALT ratio increased with increasing BNP levels. And multivariate logistic analysis showed that the AST/ALT ratio was significantly associated with a high BNP $(\geq 100 \mathrm{pg} / \mathrm{mL})$. There were 250 all-cause deaths including 79 cardiovascular deaths. Multivariate Cox proportional hazard regression analysis revealed that a high AST/ALT ratio ( $>90$ percentile) was an independent predictor of all-cause and cardiovascular mortality after adjustment for confounding factors. Kaplan-Meier analysis demonstrated that cardiovascular mortality was higher in subjects with a high AST/ALT ratio than in those without. Conclusions. The AST/ALT ratio was associated with an increase in BNP and was predictive of cardiovascular mortality in a general population. Measuring the AST/ALT ratio during routine health check-ups may be a simple and cost-effective marker for cardiovascular mortality.
\end{abstract}

\section{Introduction}

Despite advances in medicine, cardiovascular disease is still a public health problem with an increasing prevalence and high mortality [1]. It is becoming increasingly evident that it would be beneficial to identify high risk subjects for future cardiovascular disease during routine health check-ups.

Brain natriuretic peptide (BNP), which is secreted from the left ventricle through mechanical stretch, is a gold standard biomarker for the diagnosis and prognosis of heart failure [2]. Increased BNP levels are reported to be associated with all-cause and cardiovascular mortality in the general population, even in the case of a slight increase in BNP [3,4]. Although high BNP is useful for early identification of high risk subjects for cardiovascular disease, it is not routinely measured in health check-ups in general population, suggesting the importance of the surrogate marker, which is routinely measured in health check-ups.

Cardiohepatic interaction has been noted in patients with heart failure [5], atrial fibrillation [6], and myocardial infarction [7]. Although the relationship between aminotransferase levels and cardiovascular disease has become a matter of 
discussion $[8,9]$, the prognostic utility of aminotransferase has not been fully studied.

Aminotransferase is a well-known marker for liver injury and is composed of alanine aminotransferase (ALT) and aspartate aminotransferase (AST). ALT is only located in the liver, but AST is located in both the liver and myocardial tissue. Cardiac disease leads to hypoxic hepatitis, which results in a rise in serum transaminase activity caused by anoxic necrosis of the centrilobular liver cells $[10,11]$. The elevation of aminotransferase in cardiac disease is characterized by a greater increase in AST compared with ALT (due to the localization of these two enzymes). We hypothesized that the increase in AST superior to ALT is related to cardiovascular disease and it may be related to elevated BNP levels and subsequent cardiovascular mortality in health check-ups.

The purpose of the present study was to assess whether the ratio of AST to ALT (AST/ALT ratio) is associated with BNP levels and is a predictor for future cardiovascular mortality in the general population.

\section{Methods}

2.1. Ethics Statement and Study Population. The institutional ethics committee of Yamagata University School of Medicine approved the study, and all participants provided written informed consent. The procedures were performed in accordance with the Helsinki Declaration.

This study was a part of the ongoing Molecular Epidemiological Study, utilizing the resources of the Regional Characteristics of 21st Century Centers of Excellence (COE) Program and the Global COE in Japan, as previously described [12].

This study was based on a community-based annual health check-up of inhabitants from the town of Takahata in northern Japan (total population 26,026). Community members, aged $>40$ years, were invited to participate. Between June 2004 and November 2007, 3,520 subjects (1,579 men and 1,941 women) were enrolled in the study. Subjects completed a self-reported questionnaire to document their medical history, current medication use, and clinical symptoms.

Twenty six subjects were excluded due to end-stage kidney dysfunction, incomplete data, or study withdrawal.

2.2. Measurement. Hypertension was defined as systolic blood pressure $(\mathrm{BP}) \geq 140 \mathrm{mmHg}$, diastolic $\mathrm{BP} \geq 90 \mathrm{mmHg}$, or antihypertensive medication use. Diabetes mellitus was defined as fasting blood sugar (FBS) $\geq 126 \mathrm{mg} / \mathrm{dL}$, glycosylated hemoglobin A1c $\geq 6.5 \%$ (Japanese Diabetes Society value), or antidiabetic medication use.

2.3. Biochemical Markers. Blood samples for AST, ALT, and $\gamma$-glutamyl transpeptidase $(\gamma$-GTP) measurement were obtained early in the morning and the assay was performed according to the Japan Science of Clinical Chemistry (JSCC) recommendations. Blood samples were also obtained for measuring BNP. These samples were transferred to chilled tubes containing $4.5 \mathrm{mg}$ ethylenediaminetetraacetic acid disodium salt and aprotinin $(500 \mathrm{U} / \mathrm{mL})$ and centrifuged at $1,000 \times \mathrm{g}$ for 15 minutes at $4^{\circ} \mathrm{C}$. The clarified plasma samples were frozen, stored at $-70^{\circ} \mathrm{C}$, and thawed just before the assay was performed. BNP concentrations were measured using a commercially available radioimmunoassay specific for human BNP (Shiono RIA BNP assay kit, Shionogi Co. Ltd., Tokyo, Japan) $[13,14]$.

Blood samples for measuring serum heart type fatty acid binding protein (H-FABP) concentrations were drawn and centrifuged at $2,500 \times \mathrm{g}$ for $15 \mathrm{~min}$ at $4^{\circ} \mathrm{C}$ within $30 \mathrm{~min}$ of collection, and the obtained serum was stored at $-70^{\circ} \mathrm{C}$ until analysis. H-FABP levels were measured using a two-step sandwich enzyme-linked immunosorbent assay (ELISA) kit (MARKIT-M H-FABP, Dainippon Pharmaceutical Co. Ltd., Tokyo, Japan), as previously described $[15,16]$.

Estimated glomerular filtration rate (eGFR) was calculated by the modification of diet in renal disease (MDRD) equation with the Japanese coefficient $[17,18]$.

2.4. Endpoint and Median Follow-Up in Years. All subjects were prospectively followed up for a median period of 9.3 years (interquartile range, 8.3-9.4 years). The endpoint was all-cause death, which was also broken down into cardiovascular deaths and noncardiovascular deaths.

2.5. Definition of High BNP and High AST/ALT Ratio. High BNP was defined as a BNP level $\geq 100 \mathrm{pg} / \mathrm{mL}$, in accordance with previous reports and the Japanese heart failure guidelines $[19,20]$. A high AST/ALT ratio was defined as $>90$ percentile of the study population.

2.6. Statistical Analysis. All values are expressed as the mean \pm standard deviation. Continuous and categorical variables were compared with $t$-tests and chi-square tests, respectively. The Kruskal-Wallis test was used to compare the AST/ALT ratio with BNP levels. Univariate and multivariate logistic analyses were performed to determine the risk factors for a high BNP level. The receiver operating characteristics (ROC) curves for high BNP were constructed to calculate the area under the curve (AUC) for AST, ALT, and the AST/ALT ratio using the trapezoidal rule. A Cox proportional hazard analysis was performed to determine independent predictors for cardiovascular deaths, and significant predictors selected in the univariate analysis were entered into the multivariate analysis. The ROC curves for cardiovascular deaths were also illustrated and AUC was calculated to compare the prognostic capacities of biomarkers. Survival curves were constructed with the Kaplan-Meier method and compared using logrank tests. A value of $P<0.05$ was considered statistically significant. All statistical analyses were performed with a standard program package (JMP version 10, SAS Institute Inc., Cary, NC, USA).

\section{Results}

3.1. Comparison of Clinical Characteristics between Subjects with High and Low BNP Levels. The subject's baseline characteristics are shown in Table 1. There were 1,562 men and 1,932 women. The mean AST/ALT ratio was 1.18. Subjects were divided into two groups according to BNP level. Subjects with high BNP were older and had higher prevalence rates of male 
TABLE 1: Clinical characteristics of subjects with high and low BNP.

\begin{tabular}{|c|c|c|c|c|}
\hline Variables & $\begin{array}{c}\text { All subjects } \\
n=3494\end{array}$ & $\begin{array}{l}\text { Low BNP } \\
n=3347\end{array}$ & $\begin{array}{c}\text { High BNP } \\
n=147\end{array}$ & $P$ value \\
\hline Age (years) & $62 \pm 10$ & $62 \pm 10$ & $72 \pm 8$ & $<0.0001$ \\
\hline Men/women & $1562 / 1932$ & $1484 / 1863$ & $78 / 69$ & 0.0373 \\
\hline Previous CVD, $n(\%)$ & $459(13 \%)$ & $394(12 \%)$ & $65(44 \%)$ & $<0.0001$ \\
\hline Previous cancer, $n(\%)$ & $74(2.1 \%)$ & $70(2.1 \%)$ & $4(2.7 \%)$ & 0.6038 \\
\hline Previous liver disease, $n(\%)$ & $82(2.3 \%)$ & $80(2.4 \%)$ & $2(1.4 \%)$ & 0.4196 \\
\hline Smoking, $n(\%)$ & $1121(32 \%)$ & $1065(32 \%)$ & $56(38 \%)$ & 0.1106 \\
\hline Alcohol consumption, $n(\%)$ & $1454(42 \%)$ & $1390(42 \%)$ & $64(44 \%)$ & 0.6289 \\
\hline Hypertension, $n(\%)$ & $1278(37 \%)$ & $1196(36 \%)$ & $82(56 \%)$ & $<0.0001$ \\
\hline Diabetes mellitus, $n(\%)$ & $241(7 \%)$ & $224(7 \%)$ & $17(12 \%)$ & 0.0225 \\
\hline Systolic BP, mmHg & $134 \pm 16$ & $134 \pm 16$ & $138 \pm 17$ & 0.0016 \\
\hline Diastolic BP, mmHg & $80 \pm 10$ & $80 \pm 10$ & $80 \pm 10$ & 0.6310 \\
\hline HbAlc, $\%$ & $5.7 \pm 0.7$ & $5.7 \pm 0.7$ & $5.7 \pm 0.7$ & 0.1783 \\
\hline $\mathrm{FBS}, \mathrm{mg} / \mathrm{dL}$ & $95 \pm 17$ & $95 \pm 17$ & $96 \pm 15$ & 0.6199 \\
\hline $\mathrm{eGFR}, \mathrm{mL} / \mathrm{min} / 1.73 \mathrm{~m}^{2}$ & $82 \pm 16$ & $82 \pm 16$ & $71 \pm 19$ & $<0.0001$ \\
\hline Log H-FABP, ng/mL & $1.24 \pm 0.43$ & $1.23 \pm 0.43$ & $1.56 \pm 0.39$ & $<0.0001$ \\
\hline AST, IU/L & $24.6 \pm 8.3$ & $24.9 \pm 12.3$ & $26.2 \pm 9.6$ & 0.2329 \\
\hline ALT, IU/L & $22.9 \pm 11.6$ & $23.5 \pm 14.2$ & $20.9 \pm 11.3$ & 0.0273 \\
\hline$\gamma$-GTP, IU/L & $35 \pm 42$ & $36 \pm 47$ & $39 \pm 52$ & 0.3517 \\
\hline AST/ALT ratio & $1.18 \pm 0.34$ & $1.17 \pm 0.34$ & $1.38 \pm 0.43$ & $<0.0001$ \\
\hline
\end{tabular}

Data are expressed as mean \pm standard deviation or number (\%).

ALT, alanine transaminase; AST, aspartate transaminase; BNP, brain natriuretic peptide; BP, blood pressure; CVD, cardiovascular disease; eGFR, estimated glomerular filtration rate; FBS, fasting blood sugar; $\gamma$-GTP, gamma glutamyl transpeptidase; HbAlc, glycosylated hemoglobin Alc; H-FABP, heart type fatty acid binding protein.

gender, previous cardiovascular disease, hypertension, and diabetes mellitus than those with low BNP. Also, subjects with a high BNP level had higher levels of systolic BP, H-FABP, and the AST/ALT ratio and lower levels of ALT and eGFR than those with low BNP. There were no significant differences in prevalence rate of previous cancer, previous liver disease, smoking, and alcohol consumption, HbAlc, FBS, AST, and $\gamma$-GTP between subjects with high and low BNP.

3.2. The Receiver Operating Characteristic Curve for High $B N P$. The ROC curves of AST, ALT, and AST/ALT ratio were illustrated to compare the predictive capacities of high BNP. The AUC, specificity, and sensitivity for the AST/ALT ratio were $0.67,71 \%$, and $55 \%$, respectively. The AUC for the AST/ALT ratio was significantly greater than the AUC for AST and ALT, suggesting that the AST/ALT ratio improves the predictive capacity of high BNP (Figure 1).

3.3. Association between the AST/ALT Ratio and High BNP. In the simple linear analysis, there was a significant correlation between the AST/ALT ratio and BNP $(r=0.277, P<0.0001)$. As shown in Figure 2, the AST/ALT ratio was increased with increasing BNP level. To determine the risk factors for high BNP, univariate and multivariate logistic analyses were performed. In the univariate analysis, the AST/ALT ratio was significantly associated with high BNP (Table 2). In addition, age, male gender, previous cardiovascular disease, smoking, hypertension, diabetes mellitus, systolic BP, eGFR, and ALT were associated with high BNP. Multivariate logistic analysis showed that the AST/ALT ratio was significantly associated with high BNP after adjustment for age, male gender, previous cardiovascular disease, hypertension, diabetes mellitus, and eGFR (odds ratio, 1.31; 95\% confidence interval, 1.13-1.53; $P=0.0004$, Table 2).

3.4. The AST/ALT Ratio and Biomarkers. To examine the association of the AST/ALT ratio with biomarkers, subjects were divided into 6 groups according to AST/ALT ratio percentiles: $\leq 10$ percentile, $n=351 ; 10-25$ percentile, $n=$ 532; $25-50$ percentile, $n=872 ; 50-75$ percentile, $n=869$; 75-90 percentile, $n=533$; and $>90$ percentile, $n=337$. As shown in Figures 3(a) and 3(b), cardiac biomarkers such as BNP and H-FABP were significantly increased with an increasing percentile of AST/ALT ratio. Furthermore, eGFR and body mass index (BMI) were significantly decreased with an increasing percentile of AST/ALT ratio. During the follow-up period, there were 250 all-cause deaths including 79 cardiovascular deaths. As shown in Figure 4, all-cause mortality, cardiovascular mortality, and noncardiovascular mortality were higher in subjects with a $>90$ percentile of AST/ALT ratio compared to other groups.

3.5. Cardiovascular Mortality and AST/ALT Ratio. To determine whether AST/ALT ratio can predict all-cause and 
TABLE 2: Univariate and multivariate logistic analyses to predict high BNP.

\begin{tabular}{|c|c|c|c|c|c|c|}
\hline \multirow{2}{*}{ Variables } & \multicolumn{3}{|c|}{ Univariate analysis } & \multicolumn{3}{|c|}{ Multivariate analysis } \\
\hline & OR & $95 \%$ CI & $P$ value & OR & $95 \%$ CI & $P$ value \\
\hline Age (years) & 1.12 & $1.10-1.14$ & $<0.0001$ & 1.08 & $1.05-1.10$ & $<0.0001$ \\
\hline Men/women & 0.71 & $0.51-0.98$ & 0.0382 & 0.76 & $0.54-1.08$ & 0.1300 \\
\hline BMI & 0.97 & $0.92-1.02$ & 0.2485 & & & \\
\hline Previous CVD & 5.94 & $4.22-8.36$ & $<0.0001$ & 3.53 & $2.46-5.07$ & $<0.0001$ \\
\hline Previous cancer & 1.31 & $0.47-3.64$ & 0.6049 & & & \\
\hline Previous liver disease & 0.56 & $0.14-2.31$ & 0.4259 & & & \\
\hline Smoking & 1.32 & $0.94-1.85$ & 0.1116 & & & \\
\hline Alcohol consumption & 1.09 & $0.77-1.52$ & 0.6289 & & & \\
\hline Hypertension & 2.27 & $1.63-3.12$ & $<0.0001$ & 1.18 & $0.82-1.70$ & 0.3697 \\
\hline Diabetes mellitus & 1.82 & $1.08-3.08$ & 0.0245 & 1.55 & $0.88-2.72$ & 0.1258 \\
\hline Systolic BP & 1.02 & $1.01-1.03$ & 0.0016 & & & \\
\hline Diastolic BP & 1.00 & $0.99-1.02$ & 0.6309 & & & \\
\hline $\mathrm{HbAlc}^{*}$ & 1.10 & $0.96-1.27$ & 0.1785 & & & \\
\hline FBS* & 1.03 & $0.84-1.18$ & 0.6466 & & & \\
\hline $\mathrm{eGFR}^{*}$ & 0.47 & $0.37-0.57$ & $<0.0001$ & 0.71 & $0.58-0.86$ & 0.0007 \\
\hline $\mathrm{AST}^{*}$ & 1.04 & $0.97-1.12$ & 0.2523 & & & \\
\hline ALT $^{*}$ & 0.79 & $0.65-0.97$ & 0.0234 & & & \\
\hline$\gamma-\mathrm{GTP}^{*}$ & 1.04 & $0.66-1.18$ & 0.3552 & & & \\
\hline AST/ALT ratio* & 1.59 & $1.39-1.81$ & $<0.0001$ & 1.31 & $1.13-1.53$ & 0.0004 \\
\hline
\end{tabular}

ALT, alanine transaminase; AST, aspartate transaminase; BMI, body mass index; BNP, brain natriuretic peptide; BP, blood pressure; CVD, cardiovascular disease; eGFR, estimated glomerular filtration rate; FBS, fasting blood sugar; $\gamma$-GTP, gamma glutamyl transpeptidase; HbAlc, glycosylated hemoglobin Alc; OR, odds ratio.

* Per 1-SD increase.

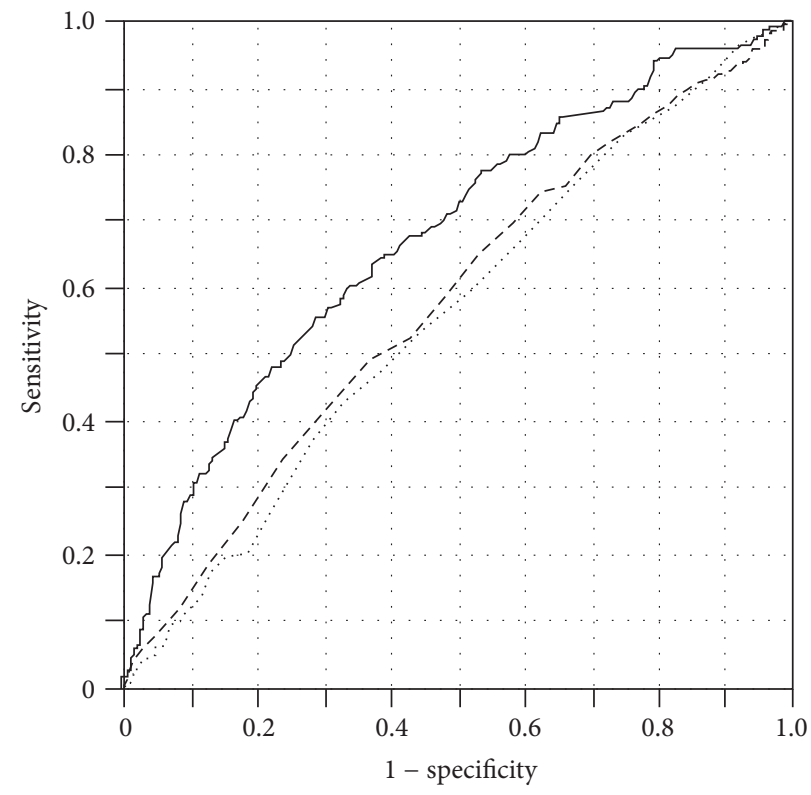

- The AST/ALT ratio; AUC $=0.67$, specificity $71 \%$, sensitivity $55 \%, P<0.0001$ versus AST and $P<0.0001$ versus ALT

- - ALT; AUC $=0.58$, specificity $63 \%$, sensitivity $49 \%$

… AST; AUC $=0.56$, specificity $67 \%$, sensitivity $43 \%$

FIGURE 1: The receiver operating characteristics curve to predict high BNP levels. The area under the curve for the AST/ALT ratio was significantly greater than for AST and ALT. ALT, alanine transaminase; AST, aspartate transaminase; BNP, brain natriuretic peptide. 


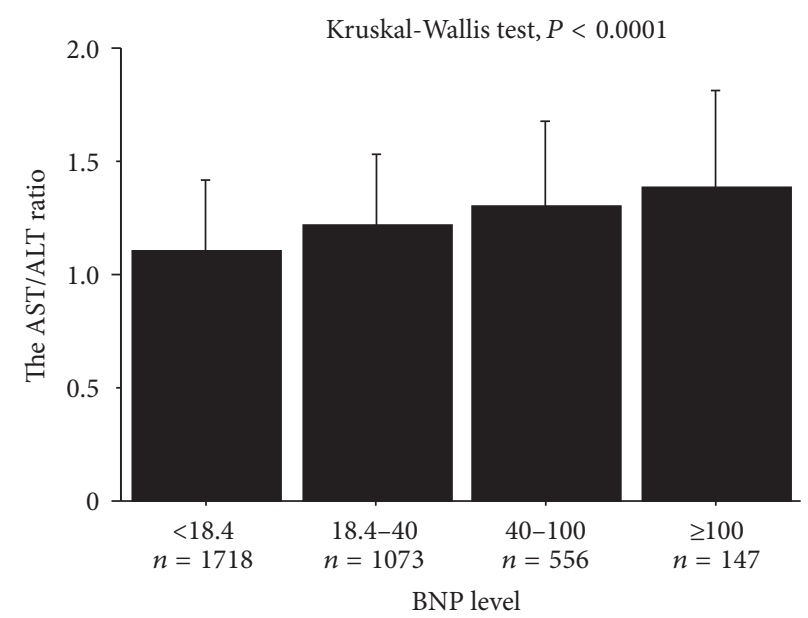

FIGURE 2: The association between the AST/ALT ratio and BNP level. The AST/ALT ratio was increased with increasing BNP levels (KruskalWallis test, $P<0.0001)$. ALT, alanine transaminase; AST, aspartate transaminase; BNP, brain natriuretic peptide.

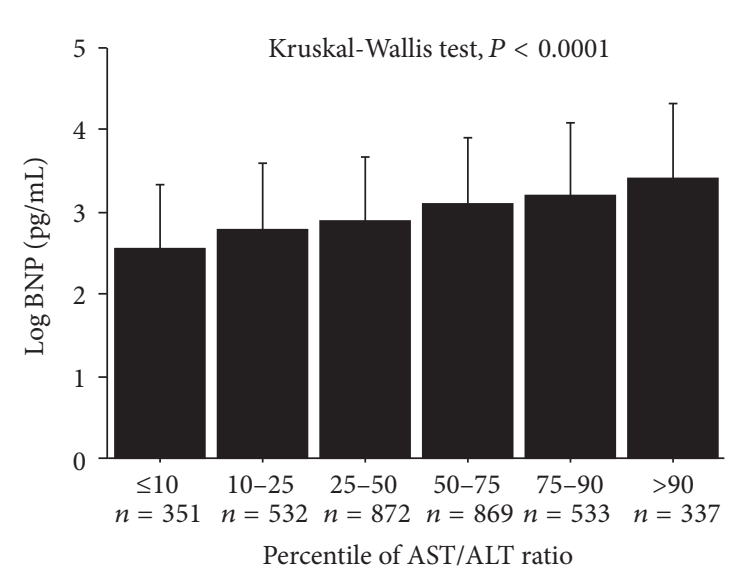

(a)

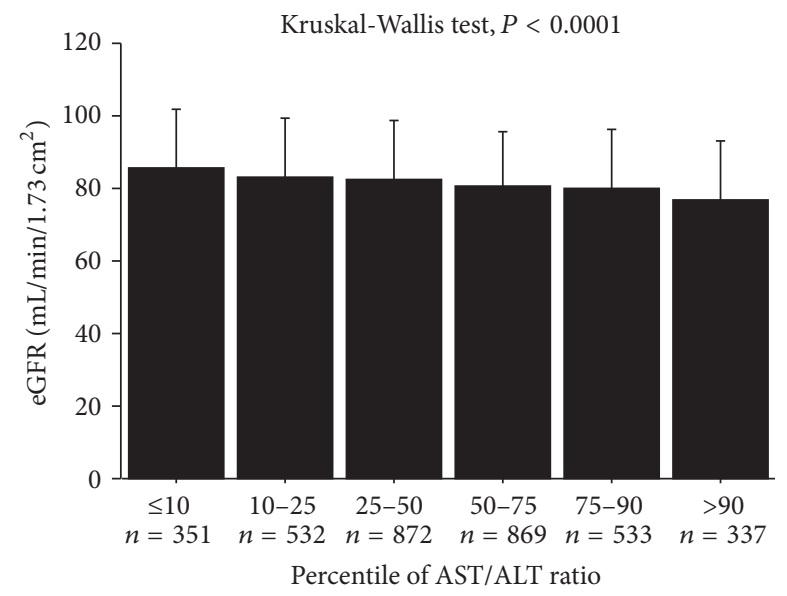

(c)

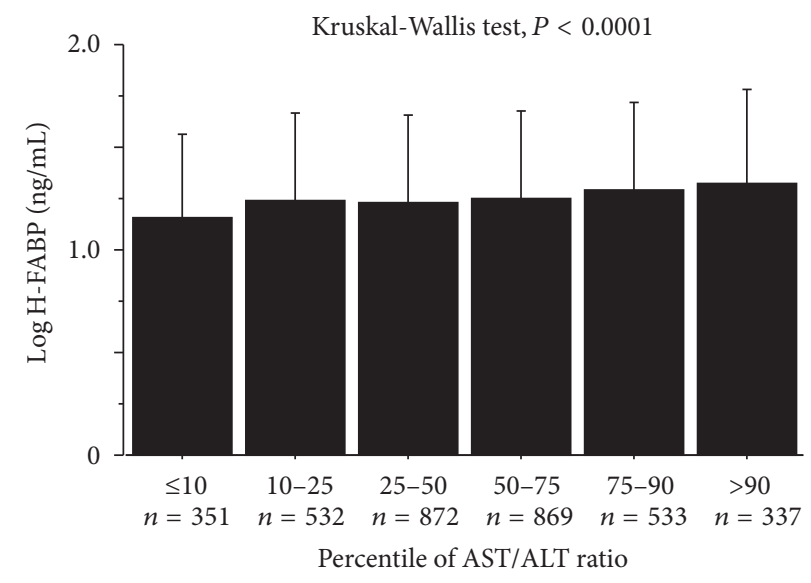

(b)

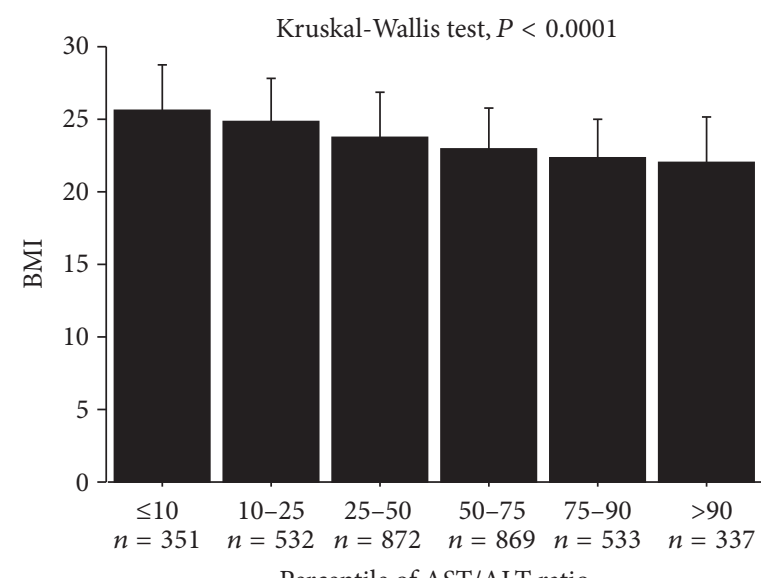

Percentile of AST/ALT ratio

(d)

FIGURE 3: The association between the AST/ALT ratio and log BNP, log H-FABP, eGFR, and BMI. ALT, alanine transaminase; AST, aspartate transaminase; BMI, body mass index; BNP, brain natriuretic peptide; eGFR, estimated glomerular filtration rate; $\mathrm{H}-\mathrm{FABP}$, heart type fatty acid binding protein. 
TABLE 3: Univariate and multivariate Cox proportional hazard regression analyses for all-cause, cardiovascular, and noncardiovascular mortality.

\begin{tabular}{|c|c|c|c|c|c|c|}
\hline \multirow{2}{*}{ Variables } & \multicolumn{3}{|c|}{ Unadjusted } & \multicolumn{3}{|c|}{ Adjusted } \\
\hline & HR & $95 \% \mathrm{CI}$ & $P$ value & HR & $95 \% \mathrm{CI}$ & $P$ value \\
\hline \multicolumn{7}{|l|}{ All-cause mortality } \\
\hline High AST/ALT ratio & 2.70 & $2.00-3.65$ & $<0.0001$ & $\begin{array}{l}1.46^{*} \\
1.43^{\#}\end{array}$ & $\begin{array}{l}1.06-2.00 \\
1.04-1.96\end{array}$ & $\begin{array}{l}0.0190 \\
0.0284\end{array}$ \\
\hline \multicolumn{7}{|l|}{ Cardiovascular mortality } \\
\hline High AST/ALT ratio & 4.35 & $2.65-7.09$ & $<0.0001$ & $\begin{array}{l}2.19^{*} \\
2.51^{\#}\end{array}$ & $\begin{array}{l}1.30-3.69 \\
1.49-4.24\end{array}$ & $\begin{array}{l}0.0031 \\
0.0006\end{array}$ \\
\hline \multicolumn{7}{|c|}{ Noncardiovascular mortality } \\
\hline High AST/ALT ratio & 2.12 & $1.44-3.13$ & 0.0001 & $\begin{array}{l}1.18^{*} \\
1.12^{\#}\end{array}$ & $\begin{array}{l}0.79-1.77 \\
0.75-1.69\end{array}$ & $\begin{array}{l}0.4109 \\
0.5800 \\
\end{array}$ \\
\hline
\end{tabular}

*Adjusted for age, gender, consumption of alcohol, smoking, hypertension, diabetes mellitus, and eGFR.

\# Adjusted for age, gender, body mass index, previous cardiovascular disease, diabetes mellitus, eGFR, and BNP.

ALT, alanine transaminase; AST, aspartate transaminase; CI, confidence interval; eGFR, estimated glomerular filtration rate; HR, hazard ratio.

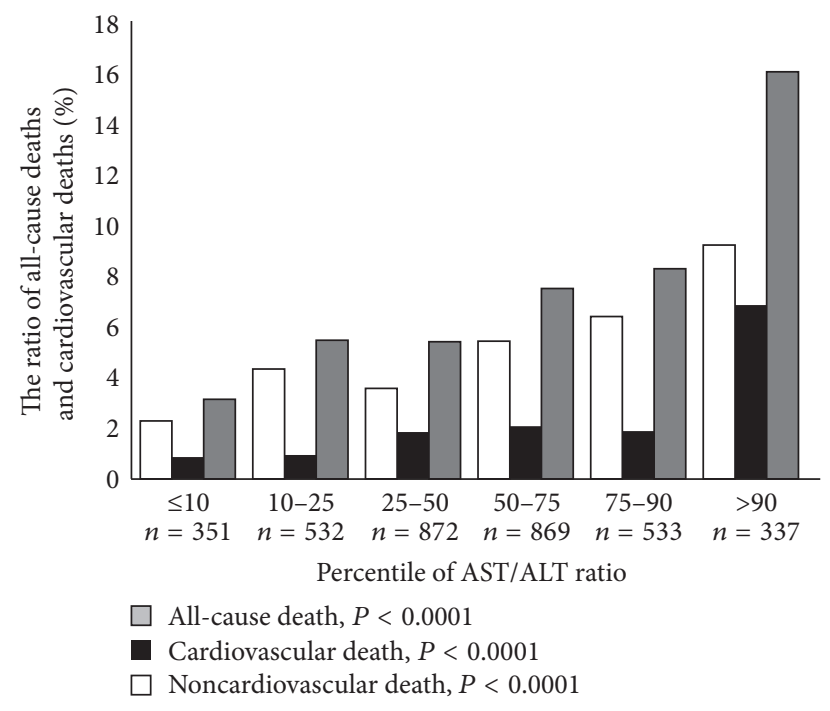

FIGURE 4: The association between the AST/ALT ratio and survival ratio. ALT, alanine transaminase; AST, aspartate transaminase.

cardiovascular mortality, we performed univariate and multivariate Cox proportional hazard regression analyses. In the univariate analysis, a high AST/ALT ratio was significantly associated with all-cause, cardiovascular, and noncardiovascular mortality (Table 3). A multivariate Cox proportional hazard regression analysis demonstrated that a high AST/ALT ratio was an independent predictor of future allcause and cardiovascular mortality, but not noncardiovascular mortality, after adjusting for confounding risk factors (Table 3).

Next, we studied the statistical interaction between AST/ALT ratio and BNP using cut-off values of these biomarkers. Multivariate Cox proportional hazard regression analysis demonstrated that subjects with abnormal levels of AST/ALT ratio $(\geq 1.6)$ and $\mathrm{BNP}(\geq 36.8 \mathrm{pg} / \mathrm{mL})$ were at significantly increased risk for cardiovascular death after adjustments for abnormal AST/ALT ratio and abnormal
TABLE 4: The area under the curves for cardiovascular deaths in general population.

\begin{tabular}{lc}
\hline & Area under the curve \\
\hline BNP & 0.73 \\
eGFR & 0.64 \\
$\gamma$-GTP & 0.55 \\
AST & 0.56 \\
ALT & 0.56 \\
AST/ALT ratio & 0.65 \\
\hline
\end{tabular}

ALT, alanine transaminase; AST, aspartate transaminase; BNP, brain natriuretic peptide; eGFR, estimated glomerular filtration rate; $\gamma$-GTP, gamma glutamyl transpeptidase.

BNP (hazard ratio, 4.18; 95\% confidence interval, 1.14-16.39; $P=0.0328)$. To compare the prognostic capacity for cardiovascular deaths, ROC analyses were performed. As shown in Table 4, AUCs in BNP and AST/ALT ratio were 0.73 and 0.65 , respectively. The AUC in AST/ALT ratio was greater than that in AST or ALT itself, indicating that transforming AST/ALT ratio improved the prognostic capacity compared to AST or ALT itself.

3.6. Comparisons of Clinical Characteristics of Subjects with a High and Low AST/ALT Ratio. As shown in Table 5, subjects with a high AST/ALT ratio were older and had higher prevalence rates of previous cardiovascular disease and lower prevalence rates of diabetes mellitus compared with those with a low AST/ALT ratio. Subjects with a high AST/ALT ratio also showed higher levels of BNP and $\mathrm{H}$ FABP and lower levels of BMI, diastolic BP, HbAlc, FBS, and eGFR than those with a low AST/ALT ratio. There were no significant differences in gender, prevalence rate of previous cancer, previous liver disease, smoking, alcohol consumption, hypertension, and systolic BP between subjects with high and low AST/ALT ratios. Kaplan-Meier analysis demonstrated that both all-cause and cardiovascular mortality were higher in subjects with a high AST/ALT ratio than in subjects with a low AST/ALT ratio (Figure 5). 
TABLE 5: Clinical characteristics between subjects with high and low AST/ALT ratio.

\begin{tabular}{|c|c|c|c|}
\hline Variables & $\begin{array}{l}\text { Low AST/ALT ratio } \\
\qquad n=3157\end{array}$ & $\begin{array}{l}\text { High AST/ALT ratio } \\
\qquad n=337\end{array}$ & $P$ value \\
\hline Age (years) & $62 \pm 10$ & $68 \pm 11$ & $<0.0001$ \\
\hline Men/women & $160 / 177$ & $1402 / 1755$ & 0.2815 \\
\hline BMI, $\mathrm{kg} / \mathrm{cm}^{2}$ & $23.7 \pm 3.1$ & $22.1 \pm 3.1$ & $<0.0001$ \\
\hline Previous CVD, $n(\%)$ & $380(12 \%)$ & $79(22 \%)$ & $<0.0001$ \\
\hline Previous cancer, $n(\%)$ & $62(2 \%)$ & $12(3 \%)$ & 0.0869 \\
\hline Previous liver disease, $n(\%)$ & $73(2 \%)$ & $9(3 \%)$ & 0.8255 \\
\hline Smoking, $n(\%)$ & $998(32 \%)$ & $123(34 \%)$ & 0.3306 \\
\hline Alcohol consumption, $n(\%)$ & $1291(41 \%)$ & $163(46 \%)$ & 0.1125 \\
\hline Hypertension, $n(\%)$ & $1134(36 \%)$ & $144(40 \%)$ & 0.1305 \\
\hline Diabetes mellitus, $n(\%)$ & $229(7 \%)$ & $12(3 \%)$ & 0.0052 \\
\hline Systolic BP, mmHg & $134 \pm 16$ & $135 \pm 17$ & 0.4902 \\
\hline Diastolic BP, mmHg & $80 \pm 10$ & $78 \pm 10$ & 0.0050 \\
\hline HbAlc, $\%$ & $5.7 \pm 0.7$ & $5.5 \pm 0.5$ & $<0.0001$ \\
\hline $\mathrm{FBS}, \mathrm{mg} / \mathrm{dL}$ & $95 \pm 17$ & $92 \pm 12$ & 0.0004 \\
\hline $\mathrm{eGFR}, \mathrm{mL} / \mathrm{min} / 1.73 \mathrm{~m}^{2}$ & $82 \pm 16$ & $77 \pm 16$ & $<0.0001$ \\
\hline Log H-FABP, ng/mL & $1.23 \pm 0.43$ & $1.34 \pm 0.45$ & $<0.0001$ \\
\hline Log BNP, pg/mL & $2.95 \pm 0.84$ & $3.42 \pm 0.90$ & $<0.0001$ \\
\hline AST, IU/L & $24.8 \pm 9.7$ & $27.4 \pm 25$ & 0.0002 \\
\hline ALT, IU/L & $24.3 \pm 13.8$ & $15.0 \pm 13.2$ & $<0.0001$ \\
\hline$\gamma$-GTP, IU/L & $36 \pm 45$ & $34 \pm 66$ & 0.3455 \\
\hline
\end{tabular}

Data are expressed as mean \pm standard deviation or number (\%).

ALT, alanine transaminase; AST, aspartate transaminase; BMI, body mass index; BNP, brain natriuretic peptide; BP, blood pressure; CVD, cardiovascular disease; eGFR, estimated glomerular filtration rate; FBS, fasting blood sugar; $\gamma$-GTP, gamma glutamyl transpeptidase; HbAlc, glycosylated hemoglobin Alc; H-FABP, heart type fatty acid binding protein.
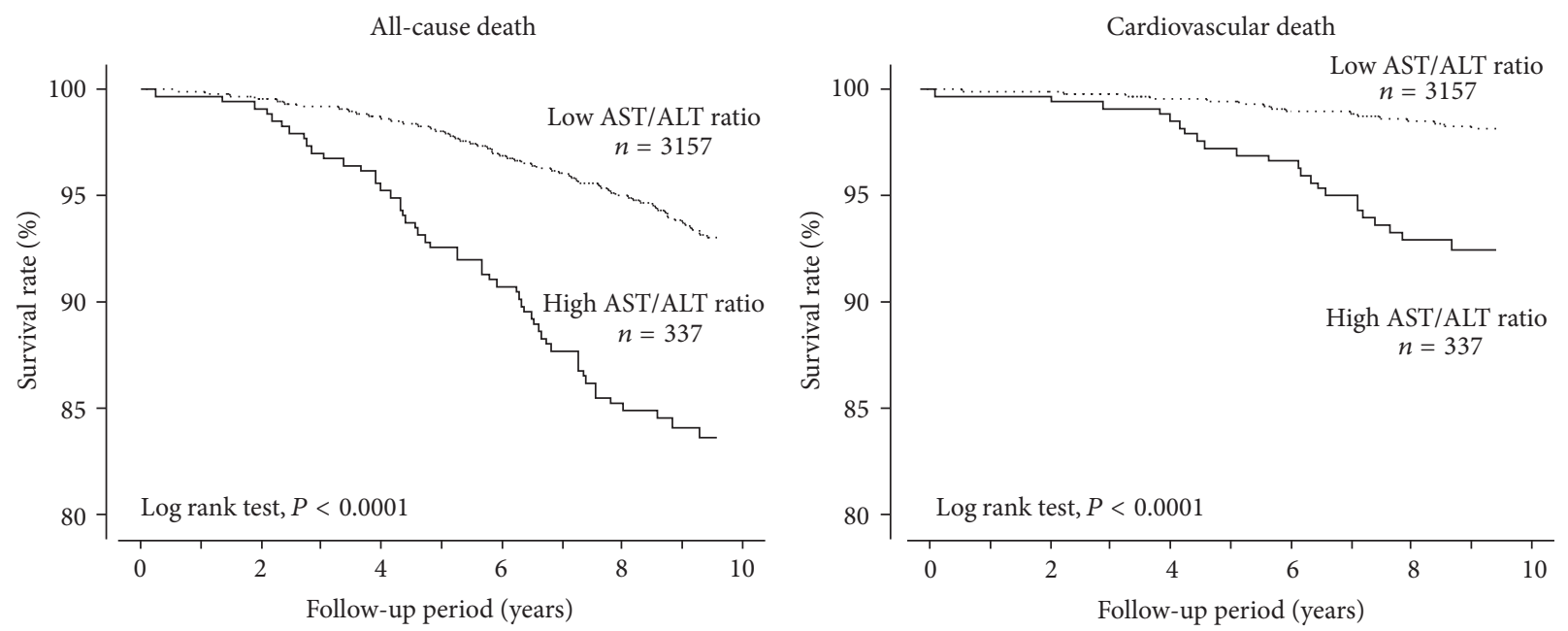

FIGURE 5: Kaplan-Meier analysis to predict all-cause and cardiovascular death between subjects with a high and low AST/ALT ratio. ALT, alanine transaminase; AST, aspartate transaminase.

\section{Discussion}

The new and novel findings from this study were as follows: (1) the AST/ALT ratio was increased with increasing BNP levels; (2) multivariate logistic analysis demonstrated that a high AST/ALT ratio was significantly associated with a high
BNP level; (3) multivariate Cox proportional hazard regression analysis demonstrated that the high AST/ALT ratio was an independent predictor of all-cause and cardiovascular mortality, but not noncardiovascular mortality; (4) subjects with a high AST/ALT ratio had higher levels of BNP and H-FABP and a lower level of eGFR; (5) Kaplan-Meier analysis 
demonstrated that all-cause and cardiovascular mortality were significantly higher in subjects with a high AST/ALT ratio than in those with a low AST/ALT ratio.

4.1. The AST/ALT Ratio Elevation in the General Population. The greater elevation of AST compared with ALT is reported to be due to induction by alcohol consumption and cardiohepatic interaction $[5-7,21,22]$. Although there were no significant differences in prevalence rates of alcohol consumption and $\gamma$-GTP levels, previous cardiovascular disease rate was higher in subjects with a high AST/ALT ratio than in those with a low AST/ALT ratio. These results suggest that an elevated AST/ALT ratio may reflect cardiac load and damage and suggest the presence of latent cardiovascular disease. Since AST is localized in myocardial tissue, a high AST/ALT ratio may represent AST leakage from the myocardial tissue secondary to myocardial damage. These findings indicated a close association of AST/ALT ratio with cardiovascular disease in the general population.

4.2. The AST/ALT Ratio and BNP Level. BNP levels are reported to be a useful biomarker for all-cause and cardiovascular mortality in the general population [3]. However, BNP levels are not routinely measured in health check-ups. On the other hand, laboratory assays for liver enzymes are common, readily available, and inexpensive. Since the AST/ALT ratio was correlated with BNP levels in the general population, it may be a significant biomarker for the identification of subjects with latent cardiovascular disease.

Serum BNP levels are affected by several factors such as BMI, kidney dysfunction, and cardiovascular disease [23, 24]. The inverse relationship between BMI and BNP is well known [23]. In addition, BNP levels are reported to increase with advancing kidney dysfunction [24]. Subjects with a high AST/ALT ratio had lower BMI and eGFR levels in the present study. These results supported our hypothesis that the AST/ALT ratio was closely associated with a high BNP level, which was associated with the presence of cardiovascular disease, low BMI, and kidney dysfunction.

4.3. The AST/ALT Ratio and Mortality. Recent studies focusing on liver enzymes demonstrated their prognostic capacity to predict cardiovascular and all-cause deaths in general populations. High aminotransferase activity is related to future mortality, whereas low ALT activity is also reported to be associated with future mortality in the general population [25-27]. Low ALT levels are reported to be associated with aging, frailty, and mortality in the elderly general population [28]. Decreased ALT is reported to result from a reduced liver size and liver blood flow [29]. In the present study, subjects with previous cardiovascular disease tended to have lower ALT levels, which may be associated with low liver blood flow as a result of latent myocardial damage. However, low ALT levels were not an independent predictor for high BNP levels. Thus, the prognostic utility of aminotransferase is limited due to its complex association with mortality in the general population. We demonstrated that the AST/ALT ratio predicts all-cause mortality and cardiovascular mortality. Since it was not significantly related to noncardiovascular mortality, the prognostic capacity of the AST/ALT ratio depended on its prediction of cardiovascular mortality. Considering the cardiohepatic interaction, the AST/ALT ratio could be a useful marker for cardiovascular mortality in the general population. We found a statistically significant interaction between AST/ALT ratio and BNP that predicted future cardiovascular deaths, indicating that the combination of AST/ ALT ratio and BNP evaluations would be useful in determining risk in the general population. Therefore, clinical perspective of the present study was that AST/ALT ratio could be the screening tool for the high BNP subjects who are at high risk for cardiovascular disease and the additional information about cardiovascular mortality to BNP in general population.

\section{Conclusions}

The AST/ALT ratio was associated with abnormal BNP levels and cardiovascular mortality in the general population. Since laboratory assays for liver enzymes are common, readily available, and inexpensive, the AST/ALT ratio could be a promising parameter to identify subjects at high risk for cardiovascular disease and mortality in the general population.

\section{Competing Interests}

The authors declare that there is no conflict of interests regarding the publication of this paper.

\section{References}

[1] NIPPON DATA80 Research Group, "Risk assessment chart for death from cardiovascular disease based on a 19-year follow-up study of a Japanese representative population," Circulation Journal, vol. 70, no. 10, pp. 1249-1255, 2006.

[2] T. Tsutamoto, A. Wada, K. Maeda et al., "Attenuation of compensation of endogenous cardiac natriuretic peptide system in chronic heart failure: prognostic role of plasma brain natriuretic peptide concentration in patients with chronic symptomatic left ventricular dysfunction," Circulation, vol. 96, no. 2, pp. 509-516, 1997.

[3] K. Kara, A. A. Mahabadi, M. H. Geisel et al., "B-type natriuretic peptide: distribution in the general population and the association with major cardiovascular and coronary events-the Heinz Nixdorf Recall Study," Clinical Research in Cardiology, vol. 103, no. 2, pp. 125-132, 2014.

[4] M. Kawai, M. Yoshimura, M. Harada et al., "Determination of the B-type natriuretic peptide level as a criterion for abnormalities in Japanese individuals in routine clinical practice: the J-ABS Multi-Center Study (Japan Abnormal BNP Standard)," Internal Medicine, vol. 52, no. 2, pp. 171-177, 2013.

[5] M. D. Samsky, C. B. Patel, T. A. DeWald et al., "Cardiohepatic interactions in heart failure: an overview and clinical implications," Journal of the American College of Cardiology, vol. 61, no. 24, pp. 2397-2405, 2013.

[6] W. Deng and L. Farricielli, "Hypoxic hepatitis and acute liver failure in a patient with newly onset atrial fibrillation and diltiazem infusion," BMJ Case Reports, vol. 2013, 2013.

[7] D. M. Lofthus, S. R. Stevens, P. W. Armstrong, C. B. Granger, and K. W. Mahaffey, "Pattern of liver enzyme elevations in acute STelevation myocardial infarction," Coronary Artery Disease, vol. 23, no. 1, pp. 22-30, 2012. 
[8] M. Monami, G. Bardini, C. Lamanna et al., "Liver enzymes and risk of diabetes and cardiovascular disease: results of the Firenze Bagno a Ripoli (FIBAR) study," Metabolism: Clinical and Experimental, vol. 57, no. 3, pp. 387-392, 2008.

[9] S. A. Porter, A. Pedley, J. M. Massaro, R. S. Vasan, U. Hoffmann, and C. S. Fox, "Aminotransferase levels are associated with cardiometabolic risk above and beyond visceral fat and insulin resistance: The Framingham Heart Study," Arteriosclerosis, Thrombosis, and Vascular Biology, vol. 33, no. 1, pp. 139-146, 2013.

[10] V. Fuhrmann, B. Jäger, A. Zubkova, and A. Drolz, "Hypoxic hepatitis-epidemiology, pathophysiology and clinical management," Wiener Klinische Wochenschrift, vol. 122, no. 5-6, pp. 129-139, 2010.

[11] J. Henrion, "Hypoxic hepatitis," Liver International, vol. 32, no. 7, pp. 1039-1052, 2012.

[12] T. Konta, Z. Hao, H. Abiko et al., "Prevalence and risk factor analysis of microalbuminuria in Japanese general population: The Takahata Study," Kidney International, vol. 70, no. 4, pp. 751756, 2006.

[13] T. Niizeki, Y. Takeishi, T. Arimoto et al., "Combination of hearttype fatty acid binding protein and brain natriuretic peptide can reliably risk stratify patients hospitalized for chronic heart failure," Circulation Journal, vol. 69, no. 8, pp. 922-927, 2005.

[14] T. Niizeki, Y. Takeishi, T. Arimoto et al., "Heart-type fatty acidbinding protein is more sensitive than troponin T to detect the ongoing myocardial damage in chronic heart failure patients," Journal of Cardiac Failure, vol. 13, no. 2, pp. 120-127, 2007.

[15] T. Arimoto, Y. Takeishi, T. Niizeki et al., "Cardiac sympathetic denervation and ongoing myocardial damage for prognosis in early stages of heart failure," Journal of Cardiac Failure, vol. 13, no. 1, pp. 34-41, 2007.

[16] Y. Otaki, T. Watanabe, H. Takahashi et al., "Association of hearttype fatty acid-binding protein with cardiovascular risk factors and all-cause mortality in the general population: The Takahata Study," PLoS ONE, vol. 9, no. 5, article e94834, 2014.

[17] S. Matsuo, E. Imai, M. Horio et al., "Revised equations for estimated GFR from serum creatinine in Japan," American Journal of Kidney Diseases, vol. 53, no. 6, pp. 982-992, 2009.

[18] T. Konta, S. Takasaki, K. Ichikawa et al., "The novel and independent association between single-point SNP of NPHP4 gene and renal function in non-diabetic Japanese population: The Takahata Study," Journal of Human Genetics, vol. 55, no. 12, pp. 791-795, 2010.

[19] H. K. Gaggin Jr. and J. L. Januzzi, "Biomarkers and diagnostics in heart failure," Biochimica et Biophysica Acta-Molecular Basis of Disease, vol. 1832, no. 12, pp. 2442-2450, 2013.

[20] I. S. Anand, L. D. Fisher, Y.-T. Chiang et al., "Changes in brain natriuretic peptide and norepinephrine over time and mortality and morbidity in the Valsartan Heart Failure Trial (Val-HeFT)," Circulation, vol. 107, no. 9, pp. 1278-1283, 2003.

[21] C. Torruellas, S. W. French, and V. Medici, "Diagnosis of alcoholic liver disease," World Journal of Gastroenterology, vol. 20, no. 33, pp. 11684-11699, 2014.

[22] A. Alonso, J. R. Misialek, M. A. Amiin et al., "Circulating levels of liver enzymes and incidence of atrial fibrillation: the Atherosclerosis Risk in Communities cohort," Heart, vol. 100, no. 19, pp. 1511-1516, 2014.

[23] R. H. Christenson, H. M. E. Azzazy, S.-H. Duh, S. Maynard, S. L. Seliger, and C. R. DeFilippi, "Impact of increased body mass index on accuracy of B-type Natriuretic Peptide (BNP) and Nterminal proBNP for diagnosis of decompensated heart failure and prediction of all-cause mortality," Clinical Chemistry, vol. 56, no. 4, pp. 633-641, 2010.

[24] S. R. Das, S. M. Abdullah, D. Leonard et al., "Association between renal function and circulating levels of natriuretic peptides (from the Dallas Heart Study)," The American Journal of Cardiology, vol. 102, no. 10, pp. 1394-1398, 2008.

[25] E. M. Koehler, D. Sanna, B. E. Hansen et al., "Serum liver enzymes are associated with all-cause mortality in an elderly population," Liver International, vol. 34, no. 2, pp. 296-304, 2014.

[26] C. E. Ruhl and J. E. Everhart, “The association of low serum alanine aminotransferase activity with mortality in the us population," American Journal of Epidemiology, vol. 178, no. 12, pp. 1702-1711, 2013.

[27] Z. Liu, H. Ning, S. Que, L. Wang, X. Qin, and T. Peng, "Complex association between alanine aminotransferase activity and mortality in general population: a systematic review and metaanalysis of prospective studies," PLoS ONE, vol. 9, no. 3, article e91410, 2014.

[28] D. G. Le Couteur, F. M. Blyth, H. M. Creasey et al., "The association of alanine transaminase with aging, frailty, and mortality," Journals of Gerontology-Series A Biological Sciences and Medical Sciences, vol. 65, no. 7, pp. 712-717, 2010.

[29] Z. Liu, S. Que, J. Xu, and T. Peng, "Alanine aminotransferase-old biomarker and new concept: a review," International Journal of Medical Sciences, vol. 11, no. 9, pp. 925-935, 2014. 


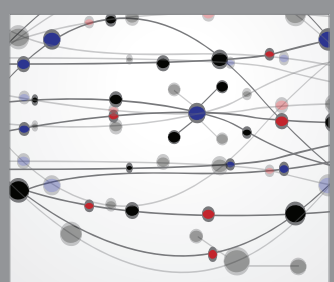

The Scientific World Journal
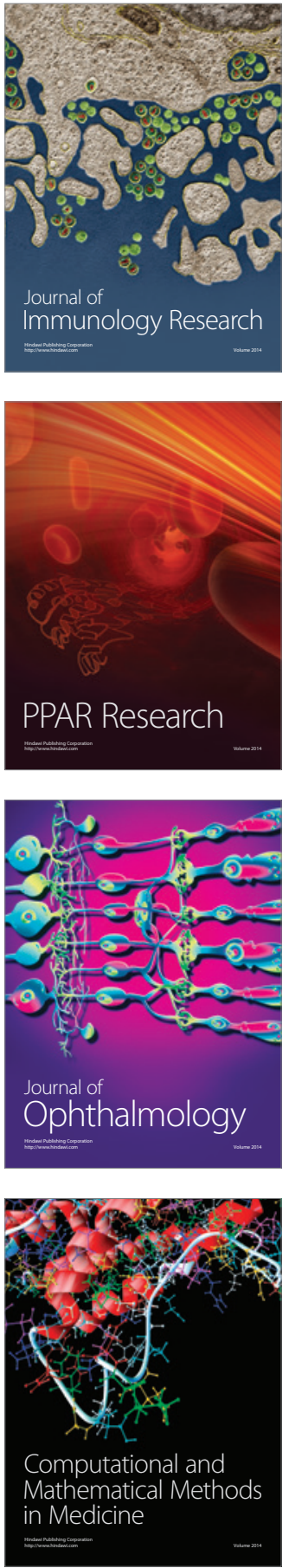

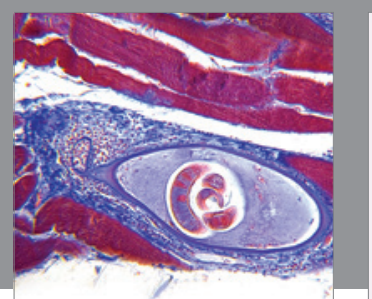

Gastroenterology Research and Practice

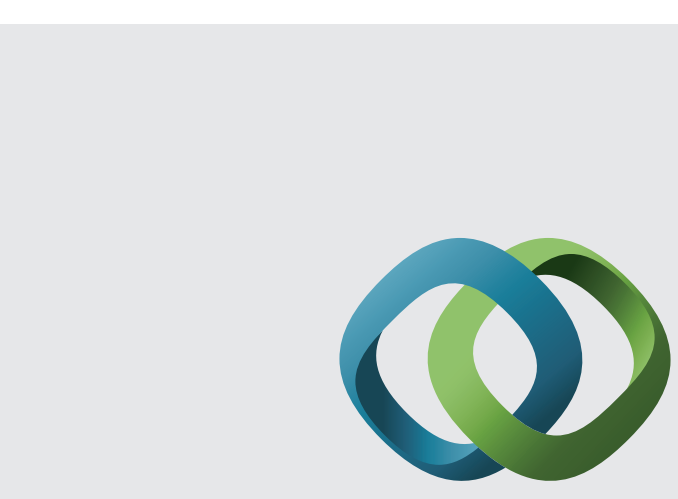

\section{Hindawi}

Submit your manuscripts at

http://www.hindawi.com
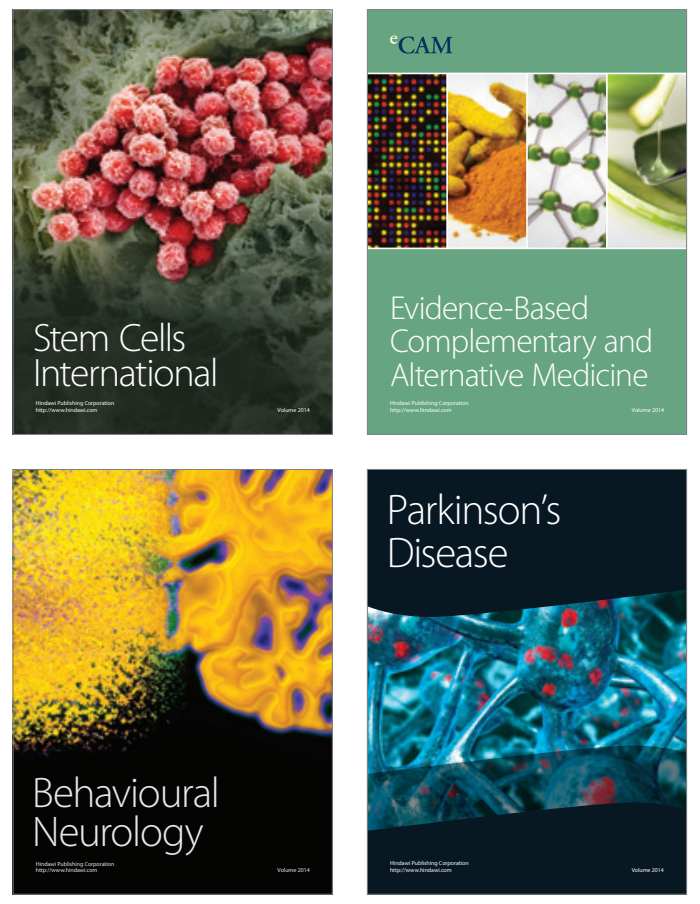
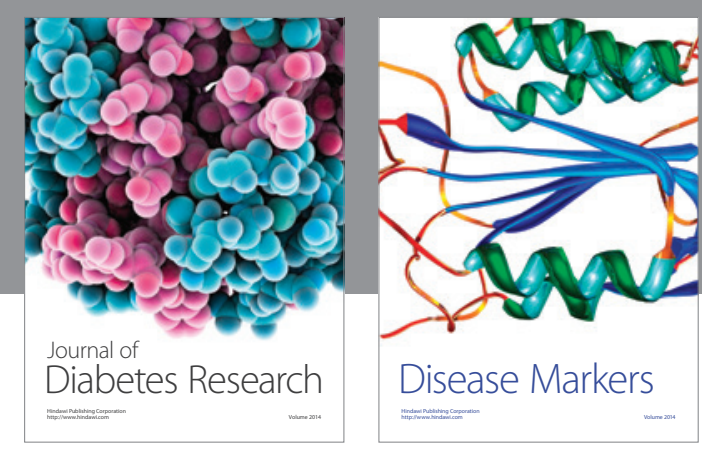

Disease Markers
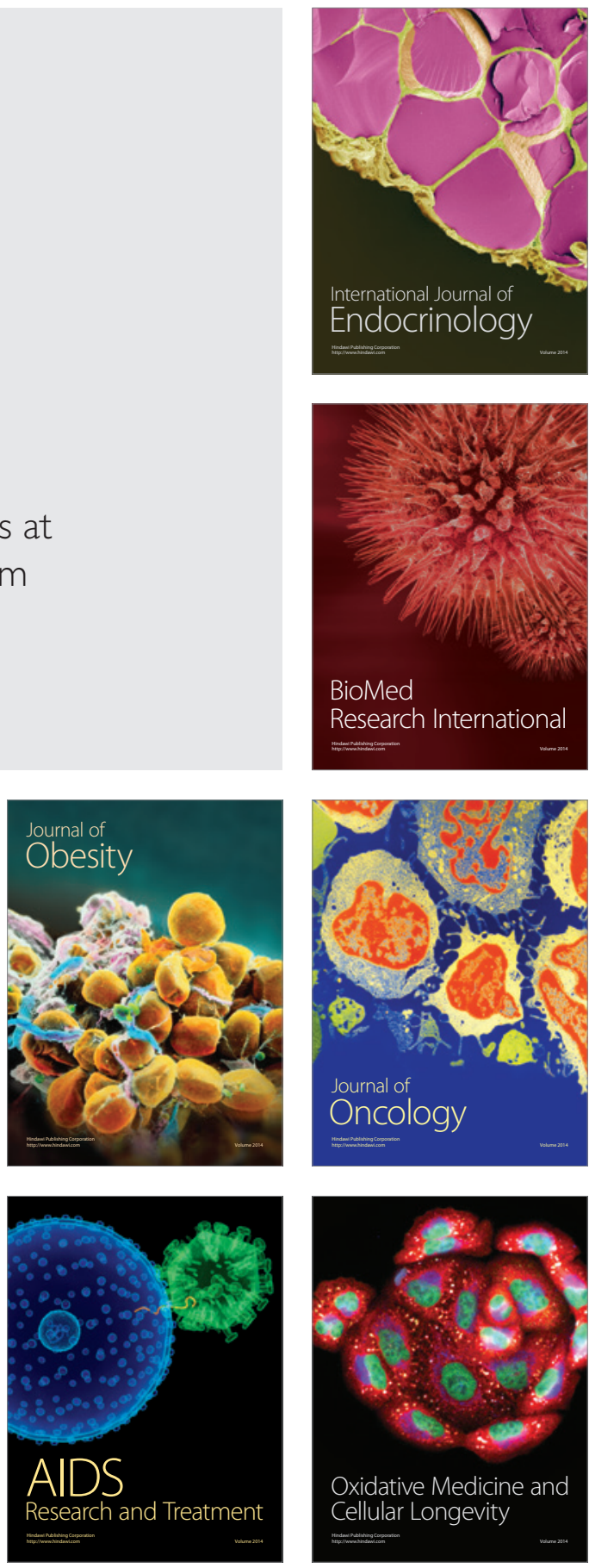